Case Report

\title{
A CASE REPORT OF HEAT STROKE : AN ICEBERG
}

\section{Pothukuchi Venkata Krishna ${ }^{1}$, Venkata Ravikumar Chepuri ${ }^{2}$, Hema Karnati ${ }^{3}$ \& Raghavareddy Yarram ${ }^{4}$ \\ ${ }^{1}$ Professor, ${ }^{2}$ Postgraduate, ${ }^{3}$ Associate Professor, ${ }^{4}$ Postgraduate, Department of Medicine, Guntur Medical College, Government General Hospital, Guntur.}

Correspondence :

Pothukuchi Venkata Krishna

57-9-15, New Postal Colony, Patamata, Vijayawada - 520 010, Andhra Pradesh. Mobile : +91 9848134088 E-mail : krishnapothukuchi@gmail.com

\begin{abstract}
:
Heat stroke was first recognized by the Romans in 24 BC. But it took until 1946 for it to be shown that heat stroke could lead to multiorgan damage with hemorrhage and necrosis in the lungs, heart, liver, kidneys, brain and gut with a 10\% to 50\% mortality rate. Patients may present with neurological impairment of varying degrees and duration, including delirium, lethargy, coma and seizures, Neurological damage is presumably attributable to metabolic disarray, cerebral edema or ischemia. Here we are presenting a case of heat stroke, with coma due to neurological involvement who recovered functionally fully though with some residual damage revealed in computerized tomogram of the brain after conservative management of heat stroke.
\end{abstract}

Keywords: Heat stroke, cerebrovenousthrombosis, neurological dysfunction, ice berg.

\section{Introduction :}

Though heat-related illnesses are known since Biblical times ${ }^{[1]}$, they are seldom addressed as a medical or health catastrophe in our society ${ }^{[2]}$. The intensity of heat stroke induced deaths are increasing with global warming and with worldwide increase in the frequency and intensity of heat waves ${ }^{[2,3]}$. In terms of clinical burden on the society and number of deaths occurring due to heat illness, hyperthermic brain injury is the third largest killer in the World after the cardiovascular and traumatic insults to the central nervous system (CNS) ${ }^{[2]}$. In spite of the seriousness of this problem, studies regarding effects of heat on the CNSare largely ignored ${ }^{[3]}$.

Heat stroke, whether classic or exertional, is a medical emergency defined by a body temperature greater than $40^{\circ} \mathrm{C}\left(104^{\circ} \mathrm{F}\right)$ that causes altered mental status and

Access this article online Quick Response Code

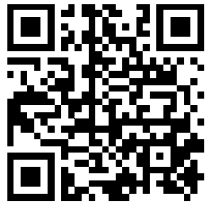
deterioration of multiple organ systems ${ }^{[4,5,6]}$. Heat stroke causes severe CNS dysfunction, e.g., delirium, convulsion and coma. More than 50 per cent of heat stroke victims die within short period despite lowering of the body temperature and therapeutic intervention. Those who survive heat stroke often show permanent neurological deficit suggesting that heat as physiological or pathological stressor considerably influences the structure and function of the nervous system ${ }^{[2]}$. The central nervous system is very sensitive to hyperthermia, causing neurologic complications due to involvement of the cerebellum, basal ganglia, anterior horn cells, and peripheral nerves ${ }^{[4,6,7]}$.

\section{Case History:}

A young unmarried female presented with history of high grade fever of two days duration associated with head ache, vomiting, reeling sensation followed by delirium and seizures after roaming in a very hot summer day. Before getting admitted in the hospital she was treated with cold and wet blankets, intravenous fluids and antipyretics for one day. There was no history of any significant illness. She was not on any medication. On examination, patient was afebrile, drowsy and incoherent. Headache and vomiting are present. There were no signs of meningeal irritation or focal neurological deficits. Papilloedema was present. Her blood sugar, renal and liver function tests and electrolytes were within normal limits. Pregnancy test was negative and urine examination was normal. Computerised Tomogram 
of brain [Figure: 1] revealed hemorrhage in left temporal region associated with edema. M agnetic resonance scan of brain [Figure:2\&3] showed hemorrhagic infarct in the left temporal region and thrombus in the left transverse and sigmoid sinus. Her connective tissue profile was normal (Antiphospholipid antibodies, Antinuclear antibodies, Anti ds-DNA were negative and, Protein C, Protein S, AntiThrombin III and Homocysteine were within normal limits).

She was treated with intravenous fluids to correct dehydration, intravenous mannitol, acetazolamide tablets, intravenous phenytoin sodium along with bladder, bowel and back care. So further tests were not done. She was treated with low molecular weight heparin initially followed by oral warfarin. Patient was improved gradually and regained full consciousness without any focal neurological cognitive deficits. She was discharged with advice to continue phenytoin sodium orally. Repeat CT scan [Figure: 4] after four months showed residual edema in the left temporal region

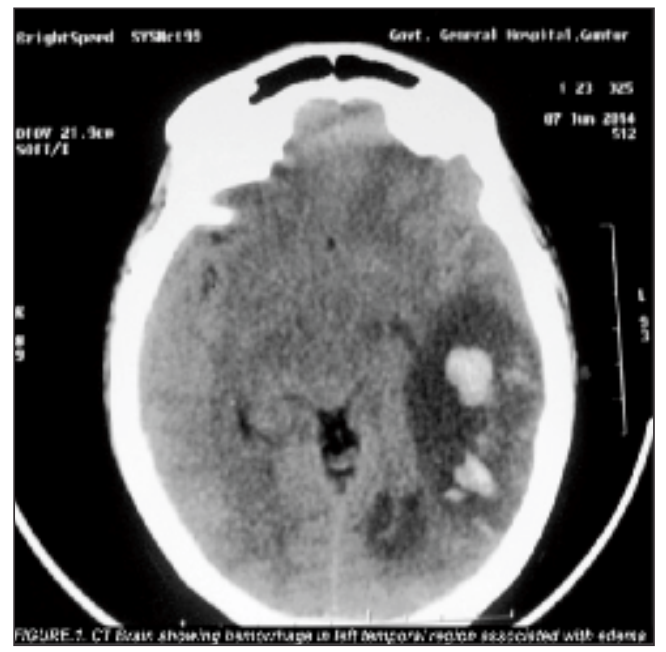

Figure 1 : CT Brain showing hemorrhage in left temporal region associated with edema.

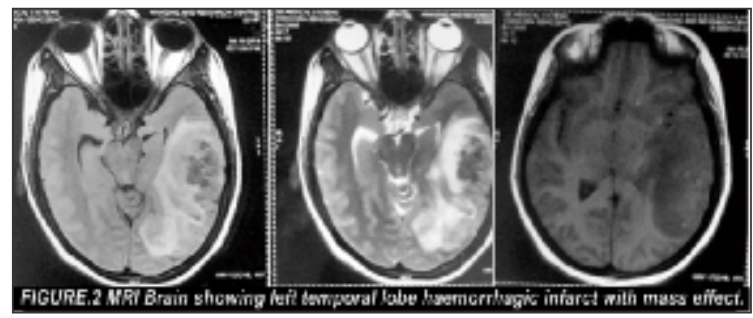

Figure 2 : MRI Brain showing haemorrhagic infarct with mass effect in the left temporal lobe.

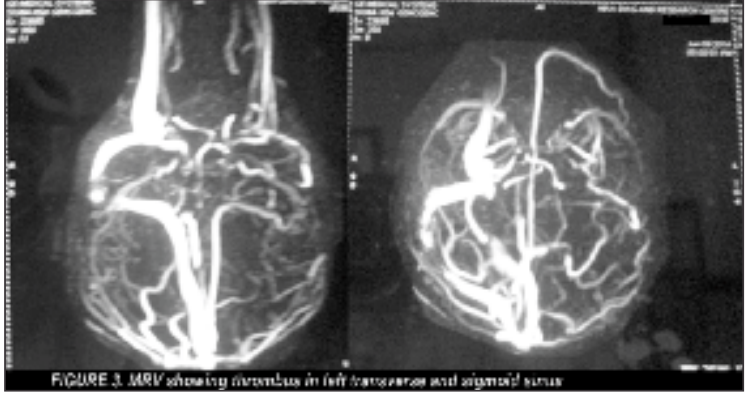

Figure 3 : MRV showing thrombus in the left transverse and sigmoid sinus.

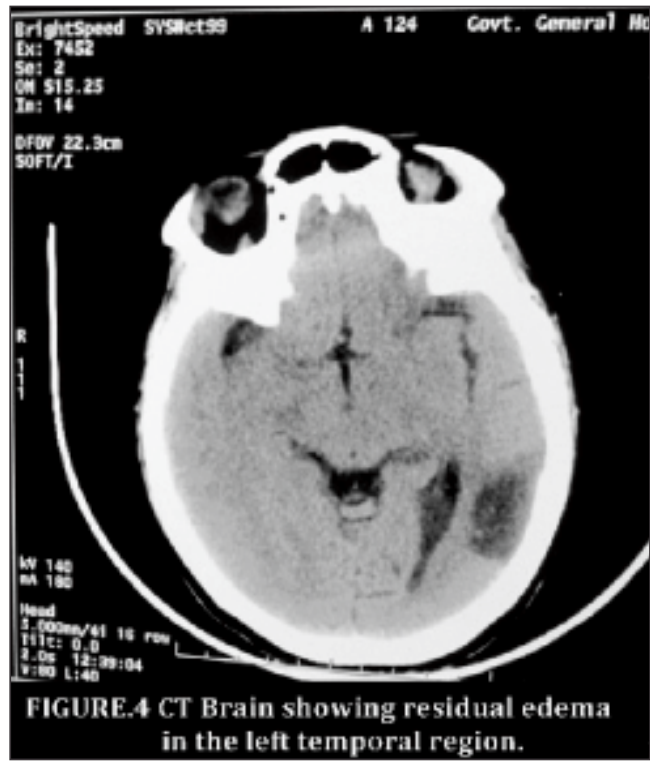

Figure 4 : CT Brain showing residual edema in the left temporal region.

\section{Discussion :}

Heat stroke is a systemic disorder and victims can therefore display a variety of symptoms and signs concordant with multiorgan dysfunction. The two cardinal features, however, are raised body temperature and neurological dysfunction. Patient may present with neurological impairment of varying degrees and duration, including delirium, lethargy, coma and seizures ${ }^{[8]}$.

Heat stroke can cause many different reactions within the body that lead to neurologic dysfunction, including decreased cerebral perfusion and aberrations in coagulation. Initially during hyperthermia, peripheral vasodilatation predominates to facilitate heat loss through the skin. To avoid a functional hypovolemia, a compensatory vasoconstriction of the splanchnic and renal 
vasculature occurs, likely causing the symptoms of nausea, vomiting, and diarrhoea. If heat stress continues, however, the compensatory vasoconstriction will eventually fail, further increasing the body temperature. Concurrently, cerebrovascular congestion and cerebral edema occur with the hyperthermia, causing an increase in intracranial pressure. Combined with a failure of splanchnic vasoconstriction and decreased mean arterial pressure, cerebral blood flow falls $s^{[4,9,10]}$. This results in cerebral ischemia. An aberration in coagulation is caused by a decrease in protein $\mathrm{C}$, protein $\mathrm{S}$, and antithrombin III, as well as alterations in vascular endothelium, creating a pattern resembling sepsis and disseminated intravascular coagulation ${ }^{[4,9]}$. This can cause hemorrhage within the brain, also resulting in neurologic dysfunction ${ }^{[4,10]}$. All of these physiologic processes occur concurrently, causing the common outcome of neuronal dysfunction ${ }^{[4]}$.

Rapid cooling is desirable and it has been shown that decreasing the body temperature below $38.9^{\circ} \mathrm{C}$ within 30 minutes of presentation improves survival ${ }^{[8,11]}$. Various methods exist to promote heat loss from the body, including cold/ ice water immersion, promotion of evaporative heat loss and the use of body cooling units. If body cooling unit is not available, keeping the patients 'wet and windy' by tepid watering of the skin and promoting air movement with fans is equally acceptable ${ }^{[8,12]}$. There are a limited number of cases in the literature that describe the CNSinjury related to heat stress ${ }^{[13]}$. Guerrero et al reported a patient with heat stress presenting with encephalopathy and bilateral cerebral, cerebellar, and thalamic lesions and intraventricular hemorrhage on $\mathrm{MRI}^{[13]}$. Carol $\mathrm{T}$ et al presented a case which demonstrates ischemic and

\section{References:}

1. Contenau G. Everyday Life in Babylon and Assyria.New York, St. Martin's Press; 1954.

2. Sharma HS. Heat-related deaths are largely due to brain damage. IndianJ Med Res 121, 2005;621-3.

3. Sharma HS, Westman J. Brain functions in hot environment.Progress in Brain Research, 115, Elsevier, Amsterdam;1998 p.1-516.

4. M cLaughlin CT, Kane AG, Auber AE: M R imaging of heat stroke: external capsule and thalamic T1 shortening and cerebellar injury. AJNR Am J Neuroradiol 2003, 24:1372-5.

5. Albukrek D, Bakon M, Moran DS, Faibel M, Epstein Y: Heat-strokeinduced cerebellar atrophy: clinical course, CT and MRI findings. Neuro-radiology 1997;39:195-7. hemorrhagic side effects of heat stroke in both the cerebral hemispheres and the cerebellum ${ }^{[4]}$. Satyendra Kumar Sonkar et al presented an unusual case of heat stroke followed by disseminated intravascular coagulation, multiple organ dysfunction with bilateral intracerebral bleed who survived with judicious management and recovered without any neurological sequeale ${ }^{[14]}$.

Our patient had high grade fever associated with head ache and vomiting followed by delirium (neurological dysfunction) after roaming outside in a hot summer day. We suspected heat stroke (smear for malarial parasite was negative, signs of meningeal irritation were absent, connective tissue profile was negative and MR Scan of brain and venogram was had shown thrombosis in left temporal and sigmoid sinus associated with left temporal hemorrhage with mass effect and herniation). Patient was treated symptomatically and conservatively. Patient improved gradually and discharged with full functional recovery. There were no case reports of heat stroke associated with thrombosis of transverse and sigmoid sinuses in the literature. Our case is also unique to have full functional recovery (though with some residual edema in the follow up CT scan) despite the severity of findings noted in the MRScan.

\section{Conclusion:}

Whenever a patient present with raised body temperature and neurological dysfunction in hot summer days, heat stroke should be suspected and treated accordingly by body cooling to minimize the morbidity and mortality. Antipyretics are of no use as thermoregulatory mechanism was failed. Whenever possible, M R Scan should be taken to assess the neurological damage..

6. Szold O, Reider-Groswasser II, Ben Abraham R, Aviram G, Segev Y, Biderman $P$, Sorkine $P$ :Gray-white matter discrimination: a possible marker for brain damage in heatstroke? EurJ Radiol 2002;43:1-5.

7. Kalita J, M isra UK. Neurophysiological studies in a patient with heat stroke.J Neurol 2001;248:993-5.

8. Grogan $\mathrm{H}$ and Hopkins PM, Heat stroke: implications for critical care and anaesthesia, Br J Anaesth 2002;88:700-7.

9. Bouchama A, Knochel JP. M edical progress: heat stroke.N Engl J M ed 2002;346:1978-88.

10. Yarbrough B, Vicario S. Heat illness. In: MarxJ, ed. Rosen's Emergency Medicine. Concepts and Clinical Practice. 5th ed. St Louis, M 0: M osby; 2002:1997-2009. 
11. Dematte JE, O'M ara K, Buescher J, Whitney CG, Forsythe S, M cNamee T, et al. Near-Fatal Heat Stroke during the 1995 Heat Wave in Chicago. Ann Intern M ed. 1998;129:173-81.

12. Slovis CM . Features and Outcomes of Classical Heat Stroke. Ann Intern Med. 1999;130:614.

13. Guerrero WR, Varghese S, SavitzS, Wu TC. : Heat stress presenting with encephalopathy and MRI findings of diffuse cerebral injury and hemorrhage. BM CNeurol. 2013;13:63.

14. Satyendra Kumar Sonkar, Deepika Soni, Gyanendra Kumar Sonkar, Heat stroke presented with disseminated intravascular coagulation and bilateral intracerebral bleed. BMJ Case Reports 2012;7-27. 